\title{
A Generalized Kundu-Eckhaus Equation With An Extra-Dispersion: Pulses Configuration
}

Hamdy. Abdel-Gawad ( $\square$ hamdyig@yahoo.com )

Cairo University Faculty of Science https://orcid.org/0000-0003-1986-2324

\section{Research Article}

Keywords: Extra dispersion, hybrid lumps, complex chirped waves, pulses characteristics

Posted Date: May 24th, 2021

DOI: https://doi.org/10.21203/rs.3.rs-386466/v1

License: (c) (i) This work is licensed under a Creative Commons Attribution 4.0 International License. Read Full License

Version of Record: A version of this preprint was published at Optical and Quantum Electronics on November 12th, 2021. See the published version at https://doi.org/10.1007/s11082-021-03224-z. 


\title{
A generalized Kundu-Eckhaus equation with an extra-dispersion: Pulses Configuration
}

\author{
${ }^{a}$ H. I. Abdel-Gawad,
}

\begin{abstract}
Raman effect is due to self-phase modulation (SPM), which is embedded in Kundu-Eckhaus equation KEE. Here, a generalized KEE is suggested by accounting for an extra dispersion. Here, we are concerned with finding the exact solutions of the proposed equation, which is done by using the unified method. In this work, we aim to show that the optical pulses OPs propagation in optical fibers may show a variety of shapes. Waves of multiple geometric shapes are observed. Among these waves, hybrid lumps, soliton, cascade, complex chirped, hybrid w-shaped, rhombus (diamond) waves and soliton modulation, which is induced by SPM. Further, the pulses intensity, frequency, wavelength, polarization, and spectral content are introduced. The results found here are of great interest in experimenting the effects of the induced dispersion on pulses configurations. Further, the colliding dynamics are inspected and as it is observed that no rogue or sharp waves formation holds, so the collision is elastic.

keywords.Extra dispersion, hybrid lumps, complex chirped waves, pulses characteristics.
\end{abstract}

${ }^{a}$ hamdig@yahoo.com

\section{Introduction}

Nonlinear Schrodinger equations NLSEs were the objective of huge number of research works in the literature. It was shown that these equations are integrable when the real and imaginary parts are taken linearly dependent [1-3]. A 
class of an infinite number of the stable bright and dark soliton, was obtained [3]. Non local NLSE was introduced in [4]. In [5\}, the generalized Darboux transformation was performed to solve NLSE. It was found that NLSEs possess an infinite number of conservation laws [6]. The solutions of NLSE coupled with Maxwell equations were considered in [6].The analytic solutions of the $(1+1)$ dimensional NLSE under periodic boundary conditions were presented in [7]. NLSEs may have a diversity of forms. They can taken to describe optical wave propagation in highly dispersive medium. It was shown that pulses propagation may lead to a varying refractive index Kerr medium [1]. Which, in turn may produce a phase shift in the pulse [8]. Mathematically, when an extra nonlinear correction to the NLSE is considered. Indeed, the equation, for nonlinear short-pulse propagation, has to include the pulse envelope derivative. It was shown that symmetric pulse will undergo an asymmetric self-phase modulation [9]. Further, the effect of self-phase modulation of a pulse propagation was analyzed in [10]. Many NLSEs contain Raman scattering effect. Which is due to SPM which may lead to an extra dispersion. Spectral compression by self-phase modulation of amplitude was investigated in [11]. The KEE .is a NLSE with Raman effect. It occupied a wide area of research in the literature [12-21]. In [12]. Explicit solutions for rogue waves.up to third order of the KEE, with quintic nonlinearity, were found. In [13]. The exact solutions of NLSEs were obtained, in the lterature, via using modified tanh coth method, extended Jacobi elliptic function expansion method, Lie symmetry analysis, conservation laws and the unified method. The long-time asymptotic behavior with nonzero boundary conditions at infinity is investigated by the steepest descent method in [14]. While, the properties of the chaotic wave fields generated are studied in [15]. Also, it was found that modulation instability results in a chaotic wave field in [16]. In [17], new complex analytical solutions in weakly nonlinear dispersive medium, by using an improved Bernoulli sub-equation method, was derived. In $\{18\}$, the Riccati-Bernoulli's sub-ODE method and Kudryashov's scheme were employed in the case of general coefficients. The variable coefficients KEE, which describes the propagation of femtosecond optical solitons, were investigated in [19]. In [20], explicit representation of the Darboux transformation was constructed to obtain analytical solutions. The Lie symmetry analysis was used to investigated optical soliton in [21].

Experimentally, the spectrometer used by Raman in (1928) was a dispersive Raman spectrometer. This suggests to use a diffraction grating spectrometer. The Raman scattering is remarkably applied in sciences [22-24]. Here, we are 
concerned of studying the behavior of solutions of the KEE with an extra dispersion. The unified method is used to find the exact solutions [25-29].

\section{Mathematical formulation}

In view of the mentioned in the above, for the well known NLSE with Raman effect reads [30-32]

$i q(x, t)_{t}+\alpha q(x, t)_{x x}+\gamma|q(x, t)|^{2} q(x, t)+i \beta\left(|q(x, t)|^{2}\right)_{x} q(x, t)=0$.

In the presence of an extra dispersion, it reads

$$
\begin{gathered}
i q(x, t)_{t}+\alpha q(x, t)_{x x}+i \delta_{1} q(x, t)_{x x x}+\gamma|q(x, t)|^{2} q(x, t) \\
+i \beta\left(|q(x, t)|^{2}\right)_{x} q(x, t)=0 .
\end{gathered}
$$

The well known KEE [32] is

$$
\begin{gathered}
i q(x, t)_{t}+\alpha q(x, t)_{x x}+\gamma|q(x, t)|^{2} q(x, t) \\
+\beta^{2}|q(x, t)|^{4} q(x, t)-2 i \beta\left(|q(x, t)|^{2}\right)_{x} q(x, t)=0 .
\end{gathered}
$$

In the presence of an extra dispersion, it reads

$$
\begin{aligned}
i q(x, t)_{t}+ & \alpha q(x, t)_{x x}+i \delta_{1} q(x, t)_{x x x}+\delta_{2} q(x, t)_{x x x x}+\gamma|q(x, t)|^{2} q(x, t) \\
& +\beta^{2}|q(x, t)|^{4} q(x, t)-2 i \beta\left(|q(x, t)|^{2}\right)_{x} q(x, t)=0,
\end{aligned}
$$

where $q$ is the complex smooth envelop function, $x$ and $t$ are spatial and temporal partial derivatives. $\alpha$,is the coefficient of the weak dispersion, $\beta$ is a real constant, $\beta^{2}$ is the quintic nonlinear coefficient, $\gamma$ is the cubic nonlinear coefficient, $\delta_{1}, \delta_{2}$ are the coefficients of the extra dispersion and the last term stands the Raman effect.

The induced dispersion in (4) have an impact on the characteristics of propagation of pulses in optical fibers. The physical parameters, wave length ,spectrum and frequency, which characterizes the pulse propagation in such a complex medium are identified [33]. To this issue, we write 


$$
q(x, t)=|q(x, t)| e^{i(\bar{k} x-\bar{\omega})},
$$

where $|q(x, t)|$ stands for the intensity, $\bar{k}$ and $\bar{\omega}$ are the wave number and frequency which are defined in

$$
\bar{k}=\frac{\int_{0}^{\infty}\left(\int_{-\infty}^{\infty}\left|q(x, t)_{x}\right| d x\right) d t}{\int_{0}^{\infty}\left(\int_{-\infty}^{\infty}|q(x, t)| d x\right) d t}, \quad \bar{\omega}=\frac{\int_{0}^{\infty}\left(\int_{-\infty}^{\infty}\left|q(x, t)_{t}\right| d x\right) d t}{\int_{0}^{\infty}\left(\int_{-\infty}^{\infty}|q(x, t)| d x\right) d t} .
$$

The spectrum is defined by

$$
Q\left(k_{0}, t\right)=\frac{1}{2 \pi} \int_{R} q(x, t) e^{-i k_{0} x} d x .
$$

We mention that when the sign of $\gamma$ is + or - , this stands to self-focusing or self-defocusing polarized pulses respectively.

Now, we find the analytic solutions of (1). To this end, we introduce a transformation that exhibits the waves produced by soliton-periodic wave collision.

$$
q(x, t)=(u(x, t)+i v(x, t)) e^{i(k x-\omega t)} .
$$

By substituting (8) into (4), we get the equations for the real and imaginary parts,

$$
\begin{aligned}
& A_{0} u+\gamma u\left(u^{2}+v^{2}\right)+\beta^{2} u\left(u^{2}+v^{2}\right)^{2}-v_{t}+4 \beta u v u_{x} \\
& +A_{1} v_{x}+4 \beta v^{2} v_{x}+A_{2} u_{x x}+A_{3} v_{x x x}+\delta_{2} u_{x x x x}=0, \\
& A_{0} v+\gamma v\left(u^{2}+v^{2}\right)+\beta^{2} v\left(u^{2}+v^{2}\right)^{2}+u_{t}-4 \beta v u v_{x} \\
& -A_{1} u_{x}-4 \beta u^{2} u_{x}+A_{2} v_{x x}-A_{3} u_{x x x}+\delta_{2} v_{x x x x}=0,
\end{aligned}
$$

where

$$
\begin{gathered}
A_{0}=k^{2} \alpha+k^{3} \delta_{1}+k^{4} \delta_{2}+\omega, A_{1}=-2 k \alpha+3 k^{2} \delta_{1} \\
+4 k^{3} \delta_{2}, A_{2}=\alpha-3 k \delta_{1}-6 k^{2} \delta_{2}, A 3:=-\delta_{1}-4 k \delta_{2}
\end{gathered}
$$

For traveling waves solutions, we use the transformations

$$
u(x, t)=U(z), v(x, t)=V(z), z=\mu x+\sigma t .
$$


By using (12), the equations. (9) and (10) become

$$
\begin{gathered}
A_{0} U+\gamma u\left(U^{2}+V^{2}\right)+\beta^{2} U\left(U^{2}+V^{2}\right)^{2}-\sigma V^{\prime}+4 \beta \mu U V U^{\prime} \\
+\mu A_{1} V^{\prime}+4 \mu \beta V^{2} V^{\prime}+\mu^{2} A_{2} U^{\prime \prime}+\mu^{3} A_{3} V^{\prime \prime \prime}+\mu^{4} \delta_{2} U^{(4)}=0, \\
A_{0} V+\gamma v V\left(U^{2}+V^{2}\right)+\beta^{2} V\left(u^{2}+v^{2}\right)^{2}+\sigma U^{\prime}-4 \mu \beta U V V \prime \\
-\mu A_{1} U^{\prime}-4 \mu \beta U^{2} U^{\prime}+\mu^{2} A_{2} V^{\prime \prime}-\mu^{3} A_{3} U^{\prime \prime \prime}+\mu^{4} \delta_{2} V^{(4)}=0 .
\end{gathered}
$$

Here,the exact solutions of (13) and (14) are found by using the unified method. By this method, the solutions of a NLPDE is expressed in a polynomial or rational solution (PSs or RSs) with appropriate auxiliary equations.

\section{PSs of (13) and (14).}

The solutions are represented in polynomial forms in an auxiliary function that satisfies an auxiliary equation,

$$
\begin{gathered}
U(z)=\sum_{i=0}^{n_{1}} h_{i} g^{i}(z), \quad V(z)=\sum_{i=0}^{n_{2}} p_{i} g^{i}(z), \\
g^{\prime}(z)^{p}=\sum_{i=0}^{p r} c_{i} g^{i}(z), p=1,2 .
\end{gathered}
$$

We mention that, in (15), $g(z)$ is the auxiliary function and the second equation is the auxiliary equation. Here, $n_{i}$ and $r$ are integers. First we consider the case $p=1$.Here, the objective is to finding $n_{i}$ and $r$. To this end, balance of the nonlinear and higher order derivative terms are invoked. Which determines $n_{i}=n_{i}(\mathrm{k}), m_{i}=m_{i}(\mathrm{k})$, which is called the balance condition. These conditions read $n_{i}=r-1, i=1,2$. To determine the value of $r$, we need to evaluate: (i) The number of equations that result from substituting (15) into. (11)-(14) and setting the coefficients o $g^{i}(z), j=0,1,2$ equal to zero (sayr $(k)$ ). (ii) The number of arbitrary paymasters $\left\{a_{j}, b_{j}, c_{j}\right\}$ in (15) (says $(k)$ ), and the higher order derivative (say $\mathrm{m}$ ). When (11)-(14) are integrable, we have $r(k)-s(k) \leq$ $m$, which results to get $\mathrm{k}$. in the present case, we have $1 \leq r \leq 3$. By the same way, the case $p=2$ is dealt with. The solutions in (15) are elementary (explicit or implicit) functions according to when or $p=2$ respectively. 


\subsection{First case when $p=2$ and $r=2$ (elliptic solutions)}

In this case, (15) becomes

$$
\begin{gathered}
U(z)=h_{1} g(z)+h_{0}, \quad V(z)=p_{1} g(z)+p_{0}, \\
g^{\prime}(z)=\sqrt{c_{4} g(z)^{4}+c_{2} g(z)^{2}+c_{0}} .
\end{gathered}
$$

By substituting (16) into (13) and (14), by setting the coefficients of $g(z)^{j}, j=$ $0,1, \ldots$, equal to zero, we get

$$
\begin{gathered}
\delta_{2}=-\frac{\beta^{2}\left(h_{1}^{2}+p_{1}^{2}\right)^{2}}{24 c_{4}^{2} \mu^{4}}, \delta_{1}=\frac{\left(h_{1}^{2}+p_{1}^{2}\right) \beta\left(k \beta\left(h_{1}^{2}+p_{1}^{2}\right)+4 c 4 \backslash \mu^{2}\right)}{6 c_{4}^{2} \mu^{4}}, h_{0}=0, p_{0}=0 \\
\sigma=\frac{2}{3 c_{4} h_{1}^{2} \mu}\left(3\left(h_{1}^{2}+p_{1}^{2}\right) \beta k^{2} h_{1}^{2}-3 c 4 h_{1}^{2} k \alpha \mu^{2}-\left(h_{1}^{2}+p_{1}^{2}\right) \beta \mu^{2}\left(c_{2} h_{1}^{2}+6 c_{4} h_{0}^{2}\right)\right. \\
\left.-12 c 4 h_{1}^{2} k^{3} \delta_{2} \mu^{2}\right), \alpha=\frac{1}{12 c_{4}^{2} \mu^{4}}\left(h_{1}^{2}+p_{1}^{2}\right)\left(3 k^{2} p_{1}^{2} \beta^{2}+24 c_{4} k \beta \mu^{2}\right. \\
+\left(5 c_{2} p_{1}^{2} \beta^{2}-12 c_{4} \gamma \mu^{2}+h_{1}^{2} \beta^{2}\left(3 k^{2}+5 c_{2} \mu^{2}\right)\right) \\
\omega=\frac{1}{24 c_{4}^{2} \mu^{4}}\left(h_{1}^{2}+p_{1}^{2}\right)\left(\left(3 k^{4} p_{1}^{2} \beta^{2}+32 c_{4} k^{3} \beta \mu^{2}\right.\right. \\
+2 k^{2}\left(5 c_{2} p_{1}^{2} \beta^{2}-12 c_{4} \gamma \mu^{2}+3\left(-3 c_{2}^{2} p_{1}^{2} \beta^{2}+4 c_{4} c_{0} p_{1}^{2} \beta^{2}\right.\right. \\
\left.+8 c_{2} c_{4} \gamma \mu^{4}+h_{1}^{2} \beta^{2}\left(3 k^{4}+10 c 2 k^{2} \mu^{2}+3\left(-3 c_{2}^{2}+4 c_{4} c_{0}\right) \mu^{4}\right)\right) .
\end{gathered}
$$

When

$$
\begin{gathered}
c_{4}=-m^{2}, c_{2}=2 m^{2}-1, c_{0}=m^{2}-1, g(z)=c n(z, m), \\
z=\mu x+\sigma t
\end{gathered}
$$

the solutions of (13) and (14) are

$$
\begin{gathered}
u(x, t)=h_{1} c n(z, m), v(x, t)=p_{1} c n(z, m), p_{1}=\sqrt{-h_{1}^{2}+\frac{2 \sqrt{6} m^{2} \mu^{2} \sqrt{-\delta_{2}}}{\beta}}, \\
k=\frac{\left(-2 m^{2} \mu^{2}\left(-2\left(h_{1}^{2}+p_{1}^{2}\right) \beta-3 m^{2} \delta_{1} \mu^{2} 2\right)\right)}{\left(h_{1}^{2}+p_{1}^{2}\right)^{2} \beta^{2}}
\end{gathered}
$$

It is worth noticing that in (19), the functions $u$ and $v$ are linearly dependent.

The results in (17)-(19) are displayed against $x$ and $t$ for $|q(x, t)|$ and $\operatorname{Req}(x, t)$ in figures 1 (i) and (ii) respectively. 
(i)

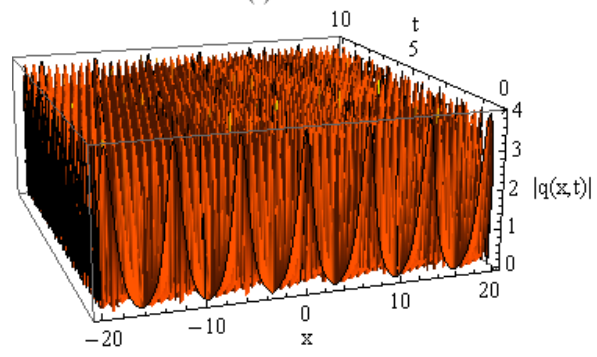

(ii)

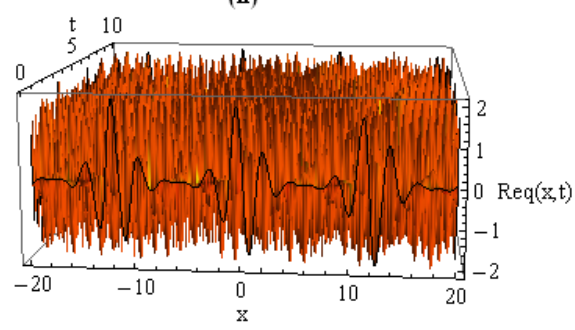

Figures1 (i) and (ii) when $m:=0.999, \delta_{2}=-0.5, \delta_{1}=2.5, h_{1}=0.6, \beta=$ $0.5, \mu=0.8, \gamma=1.1$.

Fig. (i) shows hybrid W-shaped waves while (ii) shows complex chirped waves.

\subsection{Second case when $p=2$ and $r=2$ (soliton solutions)}

In this case we consider the first equation in (16), while the auxiliary equation is

$$
g^{\prime}(z)=g(z) \sqrt{a^{2}-b^{2} g(z)^{2}} .
$$

By substituting from (16) and (20) into (13) and (14), we get

$$
\begin{gathered}
\delta_{1}=-\frac{\left(\left(2\left(h_{1}^{2}+p_{1}^{2}\right) \beta+6 b^{2} k \delta_{2} \mu^{2}\right) /\right.}{3 b^{2} \mu^{2}}, \delta_{2}=-\frac{\left(h_{1}^{2}+p_{1}^{2}\right)^{2} \beta^{2}}{24 b^{4} \mu^{4}}, h_{0}=0, p_{0}=0, \\
\sigma=-\frac{1}{6 b^{4} \mu^{3}}\left(h_{1}^{2}+p_{1}^{2}\right)\left(k^{3} p_{1}^{2} \beta^{2}-12 b^{2} k^{2} \beta \mu^{2}+k\left(5 a^{2} p_{1}^{2} \beta^{2}\right.\right. \\
\left.+12 b^{2} \gamma \mu^{2}-4 a^{2} b^{2} \beta \mu^{4}+h_{1}^{2} k \beta^{2}\left(k^{2}+5 a^{2} \mu^{2}\right)\right), \\
\alpha=\frac{1}{12 b^{4} \mu^{4}}\left(h_{1}^{2}+p_{1}^{2}\right)\left(3 k^{2} p_{1}^{2} \beta^{2}-24 b^{2} k \beta \mu^{2}+\left(5 a^{2} p_{1}^{2} \beta^{2}\right.\right. \\
\left.+12 b^{2} \gamma \mu^{2}+h_{1}^{2} \beta^{2}\left(3 k^{2}+5 a^{2} \mu^{2}\right)\right), \\
\omega=\frac{1}{24 b^{4} \mu^{4}}\left(h_{1}^{2}+p_{1}^{2}\right)\left(3 k^{4} p_{1}^{2} \beta^{2}-32 b^{2} k^{3} \beta \mu^{2}\right. \\
+2 k^{2}\left(5 a^{2} p_{1}^{2} \beta^{2}+12 b^{2} \gamma \mu^{2}\right. \\
-3 a^{2}\left(3 a^{2} p_{1}^{2} \beta^{2}+8 b^{2} \gamma \mu^{4}+h_{1}^{2} \beta^{2}\left(3 k^{4}+10 a^{2} k^{2} \mu^{2}-9 a^{4} \mu^{4}\right)\right) .
\end{gathered}
$$

Finally, we have 


$$
\begin{gathered}
u(x, t)=h_{1} g(z), \quad v(x, t)=p_{1} g(z), \\
h_{1}=\sqrt{-p_{1}^{2}+\frac{2 \sqrt{6} b^{2} \sqrt{-\delta_{2}} \mu^{2}}{\beta}}, \quad k=-\frac{\left(3 \delta_{1}+4 \sqrt{-6 \delta_{2}}\right)}{12 \delta_{2}}, \\
g(z)=\frac{\left.2 a^{2} A_{0} e^{a z}\right)}{1+a^{2} b^{2} A_{0}^{2} e^{2 a z}},
\end{gathered}
$$

where $A_{0}$ is arbitrary and $z$ is given in (18).

By using the results in (22), $\operatorname{Req}(x, t)$ is displayed against $\mathrm{x}$ and $\mathrm{t}$ in (i) (iii) in two cases.

(a) When $\beta=-0.5$.
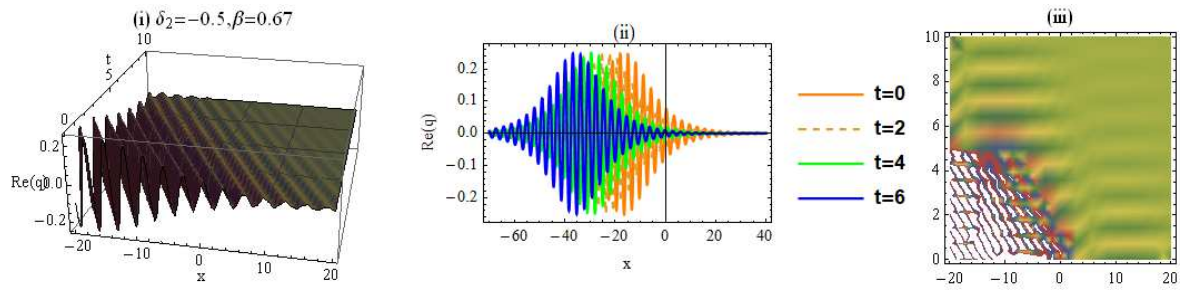

Figures 2 (i)-(iii). $\operatorname{Req}(x, t)$ is displayed when $p_{1}=-0.6, \delta_{2}=-0.5, \beta=$ $0.67, a=1.1, b=3.5, \mu=0.4, A_{0}=1.6, \gamma=0.4, \delta_{1}=1.8$.In (i) the $3 \mathrm{D}$ plot, (ii) against $x$ for different values of $t$ and (iii) the contour plot.

Figs (i) and (iii) show cascade of OPs, while (ii) shows OPs modulation which is due to SPM.

(b) When $\delta=-2.5$,

The results in $(22)$ for $\operatorname{Req}(x, t)$ is displayed in figures 3 (i) and (iii), when $\delta_{2}=-0.5$
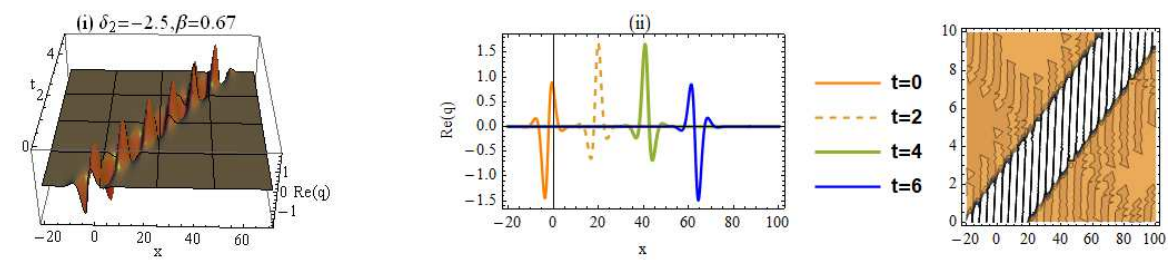

Figures 3 (i)-(iii). $\operatorname{Req}(x, t)$ is displayed for the same caption as in Figs.2 (i)-(iii).

Figs (i) and (ii) show hybrid lumps Fig. (iii) shows the density plot, where the intermediate region is bounded by the two characteristic lines $\mu x+\sigma t=$ 
$C_{1}$ and $k x-\omega t=C_{2}$. We think that outside this this region, waves propagation is chaotic.

\subsubsection{Pulses configuration.}

Here, we are concerned with investigating the wavelength, frequency and the spectrum content by using (6), (7) and (22).

Now, we consider (6), the numerical results give

\begin{tabular}{|c|c|c|c|c|c|}
\hline$\delta_{2}$ & $\bar{k}$ & $\bar{\omega}$ & $\beta$ & $\bar{k}$ & $\bar{\omega}$ \\
\hline \hline-2.5 & 0.794778 & 4.05776 & 0.5 & 0.889602 & 4.85625 \\
\hline-2 & 0.889602, & 3.90988 & 1 & 0.889602 & 2.99741 \\
\hline-1.5 & 1.0407 & 3.75348 & 1.5 & 0.889602, & 2.38699 \\
\hline-1 & 1.32425 &, 3.33526 & 2 & 0.889602 & 2.08598 \\
\hline-0.5 & 2.09102 & 1.08979 & 2.5 & 0.889602 & 1.9076 \\
\hline
\end{tabular}

Table 1 (a) and 1 (b), when

$$
\begin{gathered}
p_{1}=-0.6, a=1.1, b=2.5, \mu=0.5, A_{0}=1.6, \gamma=0.4, \delta_{1}=1.8 . \text { In } 1 \text { (a) } \\
\beta=0.7 \text {. In } 1 \text { (b) } \delta_{2}=-2.5 .
\end{gathered}
$$

By using (7), the spectrum of the OPs is shown in figures 4 (i) and (ii).
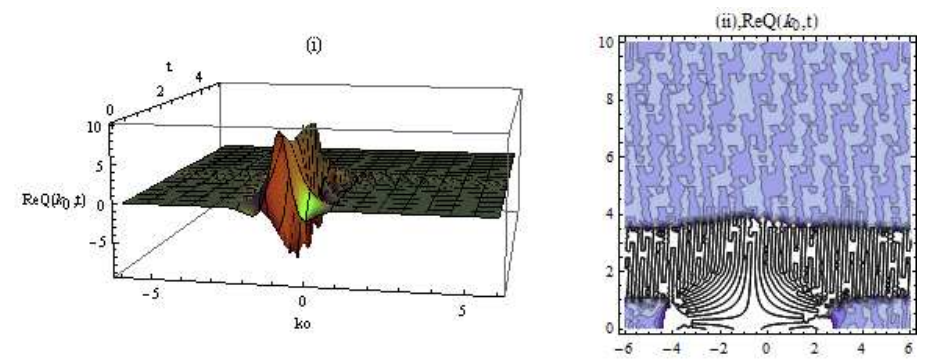

Figs. 4 (i) and (ii). With relevance to (22), the spectrum is displayed when $p_{1}=-0.6, a=1.1, b=2.5, \mu=0.5, A_{0}=1.6, \gamma=0.4, \delta_{1}=1.8, \beta=0.7, \delta_{2}=$ -2.5 . The $3 \mathrm{D}$ and contour plots are shown respectively.

Fig 4 (i) shows rhombus pulses, while (ii) shows chaotic spectrum when $t<t_{0}$. For $t>t_{0}$, the spectrum is random. This result agrees with those found in [33] for the spectra of rogue eaves.

\subsection{When $p=1$ and $r=2$.}

In this case, we consider the first equation in (16) and the auxiliary equation is 


$$
g^{\prime}(z)=c_{2} g(z)^{2}+c_{1} g(z)+c_{0} .
$$

By substituting from (16) and (23) into (13) and (14), we have

$$
\begin{gathered}
\delta_{2}=-\frac{\left(h_{1}^{2}+p_{1}^{2}\right)^{2} \beta^{2}}{24 c_{2}^{4} \mu^{4}}, \quad p_{0}=\frac{\left(-2 c_{2} h_{1} h_{0}+c_{1}\left(h_{1}^{2}+p_{1}^{2}\right)\right)}{2 c_{2} p_{1}}, \\
k_{0}=\sqrt{c_{1}^{2}-4 c_{2} c_{0}}, \quad \gamma=-\frac{\beta\left(6 c_{2}^{2} h_{0}^{3} \beta+h_{0} k_{0}^{2} p_{1}^{2} \beta+c_{2} k_{0}^{2} p_{1} \mu\right)}{6 c_{2}^{2} h_{0}}, \\
\delta_{1}=\frac{1}{12 p_{1} c_{2}^{4} \mu^{4}}\left(h_{1}^{2}+p_{1}^{2}\right) \beta\left(-c_{1} h_{1} \beta \mu\left(h_{1}^{2}+p_{1}^{2}\right)+2 h_{1}^{2} \beta\left(k p_{1}+c_{2} h_{0} \mu\right)\right) \\
+2 p_{1}\left(k p_{1}^{2} \beta+c_{2} \mu\left(h_{0} p_{1} \beta+4 c_{2} \mu\right)\right) \\
\alpha=\frac{1}{24 h_{0} p_{1}^{2} c_{2}^{4} \mu^{4}}\left(h_{1}^{2}+p_{1}^{2}\right) \beta\left(6 c_{1}^{2} h_{1}^{4} h_{0} \beta \mu^{2}+6 c_{1} h_{1}^{3} h_{0} \beta \mu\left(k p_{1}-4 c_{2} h_{0} \mu\right)\right. \\
+h_{1}^{2} h_{0} \beta\left(-6 k^{2} p_{1}^{2}-12 c_{2} h_{0} k p_{1} \mu+\left(24 c_{2}^{2} h_{0}^{2}+\left(6 c_{1}^{2}+5 k_{0}^{2}\right) p_{1}^{2}\right) \mu^{2}\right) \\
6 c_{1} h_{1} h_{0} p_{1} \mu\left(k p_{1}^{2} \beta+4 c_{2} \mu\left(-h_{0} p_{1} \beta+c 2 \mu\right)\right)-p_{1}\left(4 c_{2} k_{0}^{2} p_{1}^{2} \mu^{3}\right. \\
+h_{0}\left(6 k^{2} p_{1} \beta+48 c_{2}^{2} k p_{1} \mu^{2}-k_{0}^{2} p 1^{\wedge} 3 \beta \mu^{2}\right) \\
\left.\left.+12 h_{0}^{2}\left(c_{2} k p_{1}^{2} \beta \mu+4 c_{2}^{3} \mu^{3}\right)\right)\right)
\end{gathered}
$$

where there two very lengthy equations for $\sigma$ and $\omega$ which will not produced here.Finally, the solutions are

$$
\begin{gathered}
g(z)=\frac{-c_{1}-k_{0} \operatorname{Tanh}\left(\frac{1}{2} k_{0}\left(z+A_{0}\right)\right)}{2 c_{2}}, \quad p_{1}=\sqrt{-h_{1}^{2}+\frac{c_{2}^{2} \sqrt{-6 \delta_{2}} \mu^{2}}{\beta}}, \\
u(x, t)=h o+h_{1} \frac{-c_{1}-k_{0} \operatorname{Tanh}\left(\frac{1}{2} k_{0}\left(z+A_{0}\right)\right)}{2 c_{2}}, \\
v(x, t)=h_{1}\left(c_{1} h_{1}-2 c_{2} h_{0}\right) \beta+k o\left(h_{1}^{2} \beta-2 c_{2}^{2} \sqrt{-6 \delta_{2}} \mu^{2}\right) \operatorname{Tanh}\left(\frac{1}{2} k_{0}\left(z+A_{0}\right)\right) .
\end{gathered}
$$

where $A_{0}$ is arbitrary and $k_{0}$ is given in (24).

By using the results in $(25), \operatorname{Re}(x, t)$ is displayed against $x$ and $t$ in figures 5 (i) and (ii). 

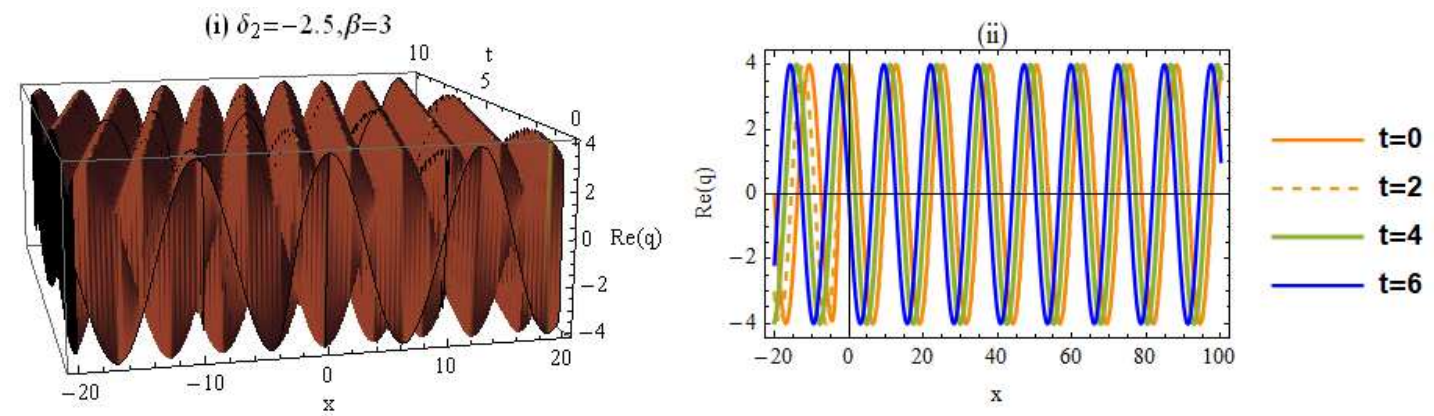

Figs.5 (i) and (ii). When $h_{1}=0.6, \delta_{2}=-2.5, \beta=3 ; c_{0}=1.6, c_{1}=3.1, c_{2}=$ $-1.5, \mu=0.5, A_{0}=1.6, \delta_{1}=1.8, k=0.5, h_{0}=2.5$.

Fig. (i) shows periodic rhombus (diamond) waves in space and time, while (ii) shows periodic waves in space and time.

It is worthy to mention that in the previous cases the solutions $u(x, t)$ and $v(x, t)$ are linearly dependent.

\subsection{When $p=1$ and $r=3$.}

In this case

$$
\begin{gathered}
U(z)=h_{2} g(z)^{2}+h_{1} g(z)+h_{0}, \quad V(z)=p_{2} g(z)^{2}+p_{1} g(z)+p_{0}, \\
g^{\prime}(z)=c_{3} g(z)^{3}+c_{2} g(z)^{2}+c_{1} g(z)+c_{0} .
\end{gathered}
$$

For linearly dependent solutions, we take $h_{1}=\frac{h_{2} p_{1}}{p_{2}}, h_{0}=\frac{h_{2} p_{0}}{p_{2}}$.

By substituting from (26) into (13) and (14), we get 


$$
\begin{gathered}
\delta_{2}=-\frac{\left(h_{2}^{2}+p_{2}^{2}\right)^{2} \beta^{2}}{384 c_{3}^{4} \mu^{4}}, \quad c_{1}=-\frac{2 p_{2}}{p_{1}}, c_{0}=-1-\frac{c_{3} p_{1}^{3}}{4 p_{2}^{3}}, c_{2}=\frac{3 c_{3} p_{1}}{2 p_{2}}, \\
\gamma=-\frac{1}{32 c_{3}^{2} p_{1}^{2} p_{2}^{4}}\left(h_{2}^{2}+p_{2}^{2}\right) \beta^{2}\left(64 p_{2}^{6}+32 c_{3} p_{1} p_{2}^{3}\left(p_{1}^{2}+2 p_{2} p_{0}\right)\right. \\
\left.+c_{3}^{2}\left(5 p_{1}^{6}+8 p_{1}^{4} p_{2} p_{0}+32 p_{1}^{2} p_{2}^{2} p_{0}^{2}\right)\right), \\
\delta_{1}=\frac{1}{384 c_{3}^{4} h_{2} p_{1} p_{2} \mu^{4}}\left(h_{2}^{2}+p_{2}^{2}\right) \beta\left(4 h_{2}^{3} k p_{1} p_{2} \beta-5 h_{2}^{2}\left(8 p_{2}^{3}\right.\right. \\
\left.+c_{3}\left(p_{1}^{3}+8 p_{1} p_{2} p_{0}\right)\right) \beta \mu-5 p_{2}^{2}\left(8 p_{2}^{3}+c_{3}\left(p_{1}^{3}+8 p_{1} p_{2} p_{0}\right)\right) \beta \mu \\
\left.+4 h_{2} p_{1} p_{2}\left(k p_{2}^{2} \beta+16 c_{3}^{2} \mu^{2}\right)\right), \\
\alpha=\left(h_{2}^{2}+p_{2}^{2}\right) \beta\left(60 k p_{1} p_{2}^{5}\left(8 p_{2}^{3}+c_{3}\left(p_{1}^{3}+8 p_{1} p_{2} p_{0}\right)\right) \beta \mu\right. \\
+12 h_{2}^{2} p_{1} p_{2}\left(8 p_{2}^{3}+c_{3}\left(p_{1}^{3}+8 p_{1} p_{2} p_{0}\right)\right) \mu\left(5 k p_{2}^{2} \beta-32 c_{3}^{2} \mu^{2}\right) \\
+h_{2}^{3} \beta-24 k^{2} p_{1}^{2} p_{2}^{4}+\left(1472 p_{2}^{6}+48 c_{3} p_{1} p_{2}^{3}\left(7 p_{1}^{2}+64 p_{2} p_{0}\right)+\right. \\
\left.\left.3 c_{3}^{2}\left(5 p_{1}^{6}+128 p_{1}^{4} p_{2} p_{0}+512 p_{1}^{2} p_{2}^{2} p_{0}^{2}\right)\right) \mu^{2}\right)+h_{2} p_{2}^{2}\left(-24 k 2 p_{1}^{2} p_{2}^{4} \beta\right) \\
-768 c_{3}^{2} k p_{1}^{2} p_{2}^{2} \mu^{2}+\left(1472 p_{2}^{6}+48 c_{3} p_{1} p_{2}^{3}\left(7 p_{1}^{2}+64 p_{2} p_{0}\right.\right. \\
\left.\left.\left.+3 c_{3}^{2}\left(5 p_{1}^{6}+128 p_{1}^{4} p_{2} p_{0}+512 p_{1}^{2} p_{2}^{2} p_{0}^{2}\right)\right) \beta \mu^{2}\right)\right), \quad c_{3}=-4\left(\frac{p_{2}}{p_{1}}\right)^{3},
\end{gathered}
$$

together with an equation for $\omega$ which is very lengthy to be produced here.

Finally, the solutions are

$$
\begin{gathered}
g(z)=\frac{\left(-e^{\frac{2 p_{2} z}{p_{1}}} p_{1}+4 A_{0} p_{1} p_{2}+\sqrt{\left.e^{\frac{2 p_{2} z}{p_{1}}} p_{1}^{2}\left(e^{\frac{2 p_{2} z}{p_{1}}}-4 A o p 2\right)\right)}\right.}{2 p_{2} e^{\frac{2 p_{2} z}{p_{1}}}-4 A_{0} p_{2}}, \\
u(x, t)=\frac{h_{2}\left(e^{\frac{2 p_{2} z}{p_{1}}} p_{0}+A_{0}\left(p_{1}^{2}-4 p_{2} p_{0}\right)\right)}{p_{2}\left(e^{\frac{2 p_{1} z}{p_{1}}}-4 A_{0} p_{2}\right)}, \quad v(x, t)=\frac{\left(A_{0} p_{1}^{2}+e^{\frac{2 p_{2} z}{p_{1} z}} p_{\left.o-4 A_{0} p_{2} p_{0}\right)}\right.}{e^{\frac{2 p_{2} z}{p_{1}}}-4 A_{0} p_{2}}, \\
z=\mu x+\sigma t .
\end{gathered}
$$

By using the results in (28), Req $(x, t)$. is displayed against $\mathrm{x}$ and $\mathrm{t}$ in figures 6 (i) and (ii).
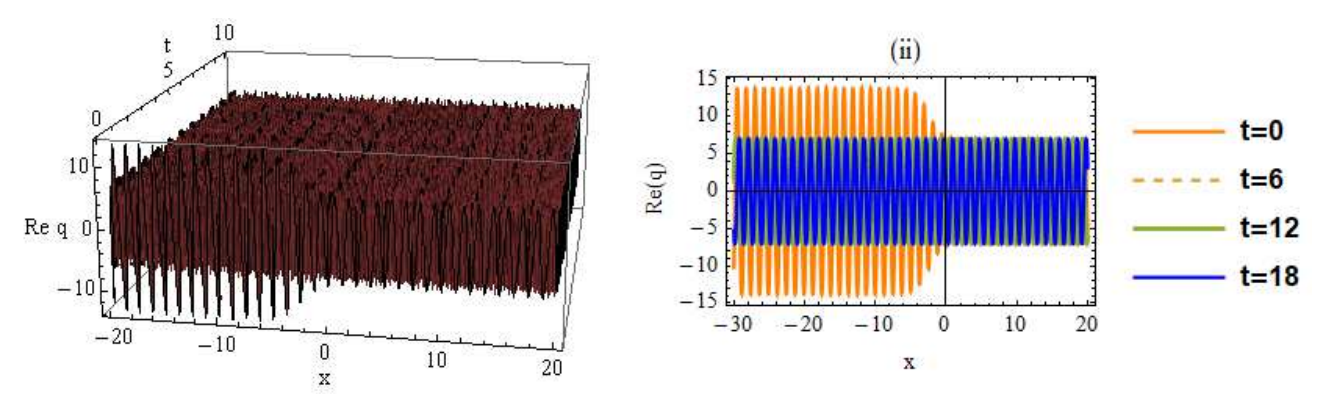

Figures 6 (i) and (ii0. When $A_{0}=1.1, p_{1}=0.9, \delta_{2}=-2.5, \beta=3, p_{2}=$ 
$-1.7, \mu=0.3$.

Fig. (i) shows hybrid chirped waves on $x<0$ and periodic M-shaped waves on $x>0$, while (ii) shows solitary wave modulation, which is due to SPM.

\section{RSs of (13) and (14).}

(i) When $k=1$,we write the solution in the form

$$
U(z)=\frac{h_{1} g(z)+h_{0}}{s_{1} g(z)+s_{0}}, \quad V(z)=\frac{p_{1} g(z)+p_{0}}{s_{1} g(z)+s_{0}}, \quad g^{\prime}(z)=c_{1} g(z)+c_{0}
$$

For linearly dependent solutions we take $p_{0}=\frac{p_{1} h_{0}}{h_{1}}$. By substituting from (29) into (13) and (14), we have

$$
\begin{aligned}
& \delta_{2}=-\frac{\left(h_{1}^{2}+p_{1}^{2}\right)^{2}\left(h_{0} s_{1}-h_{1} s_{0}\right)^{4} \beta^{2}}{24\left(c_{1} h_{1} s_{1} s_{0} \mu\right)^{4}}, \gamma=-\frac{\left(h_{1}^{2}+p_{1}^{2}\right)\left(\left(h_{0} s_{1}\right)^{2}+\left(h_{1} s_{0}\right)^{2}\right) \beta^{2}}{2\left(h_{1} s_{1} s_{0}\right)^{2}} \\
& c_{0}=0, k:=-\frac{c_{1} h_{1} \mu}{p_{1}}, \quad \delta_{1}=-\frac{\left(h_{1}^{2}+p_{1}^{2}\right) \beta\left(h_{0} s_{1}-h_{1} s_{0}\right)^{2}}{12 c_{1}^{3} h_{1}^{5} p_{1} s_{1}^{4} 4 s_{0}^{4} \mu^{3}} \\
& \left(5 h_{0}^{2} p_{1}^{4} s_{1}^{2} \beta-4 h_{1}^{5} h_{0} s_{1} s_{0} \beta+2 h_{1}^{6} s_{0}^{2} \beta+h_{1}^{2} p_{1}^{2}\left(7 h_{0}^{2} s_{1}-5 p_{1}^{2} s_{0}^{2}\right) \beta\right. \\
& \left.h_{1}^{4}\left(2 h_{0}^{2} s_{1}^{2}-3 p_{1}^{2} s_{0}^{2}\right) \beta-4 h_{1}^{3} p_{1} s_{1} s_{0}\left(h_{0} p_{1} \beta+2 c_{1} s_{1} s_{0} \mu\right)\right), \\
& \omega=\frac{\left(h_{1}^{2}+p_{1}^{2}\right) \beta}{24 h_{1}^{2} p_{1}^{4} s_{1}^{4} s_{0}^{4}}\left(-44 h_{1} h_{0}^{3} p_{1}^{4} s_{1}^{3} s_{0} \beta+78 h_{1}^{2} h_{0}^{2} p_{1}^{4} s_{1}^{2} s_{0}^{2} \beta-12 h_{1}^{7} h_{0} s_{1} s_{0}^{3} \beta\right. \\
& +3 h_{1}^{8} s_{0}^{4} \beta+2 h_{1}^{6} s_{0}^{2}\left(9 h_{0}^{2} s_{1}^{2}-20 p_{1}^{2} s_{0}^{2} \beta-\right. \\
& 3 h_{0}^{2} p_{1}^{4} s_{1}^{2}\left(h_{0}^{2} s_{1}^{2}-8 p_{1}^{2} s_{0}^{2}\right) \beta+4 h_{1}^{3} h_{0} p_{1} s_{1} s_{0}\left(-14 h_{0}^{2} p_{1} s_{1}^{2} \beta+9 p_{1}^{3} s_{0}^{2} \beta+\right. \\
& 4 c_{1} h_{0} s_{1}^{3} s_{0} \mu-4 h_{1}^{5} s_{1} s_{0}\left(3 h_{0}^{3} s_{1}^{2} \beta-6 h_{0} p_{1}^{2} s_{0}^{2} \beta+20 c_{1} p_{1} s_{1} s_{0}^{3} \mu\right)+ \\
& \left.h_{1}^{4}\left(3 h_{0}^{4} s_{1}^{4} \beta+72 h_{0}^{2} p_{1}^{2} s_{1}^{2} s_{0}^{2} \beta-43 p_{1}^{4} s_{0}^{4} \beta+64 c_{1} h_{0} p_{1} s_{1}^{3} s_{0}^{3} \mu\right)\right)
\end{aligned}
$$

together with a lengthy equation for $\sigma$, which will not produced here.

Finally, we have

$$
\begin{gathered}
g(z)=A_{0} e^{c_{1} z}, \quad u(x, t)=\frac{h_{0}+h_{1} g(z)}{s_{0}+s_{1} g(z)}, \quad v(x, t)=\frac{\left(h_{0}+h_{1} g(z)\right) p_{1}}{\left(s_{0}+s_{1} g(z)\right) h_{1}} \\
p_{1}=\frac{h_{1}}{\left(h_{0} s_{1}-h_{1} s_{0}\right)} \sqrt{-\left(h_{0} s_{1}-h_{1} s_{0}\right)^{2}+\frac{2 c_{1}^{2} s_{1}^{2} s_{0}^{2} \mu^{2} \sqrt{-6 \delta_{2}}}{\beta}} .
\end{gathered}
$$


The results in (31) are used to display $\operatorname{Req}(x, t)$ against $x$ and $t$ in figures 7 (i0, (ii) and (iii) by varying the parameters $\delta_{2}$ and $\beta$.
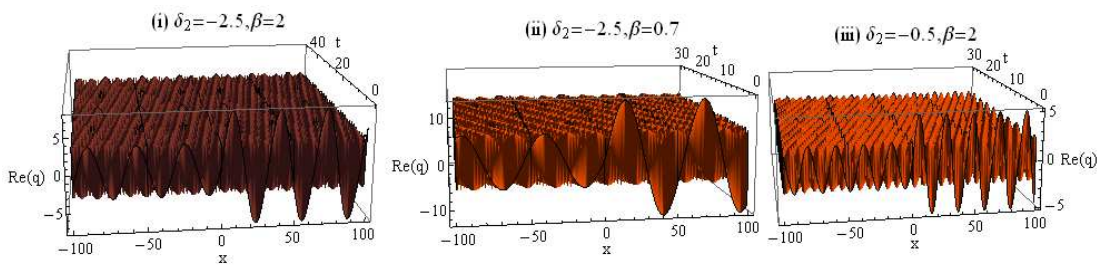

Figures 7 (i)-(iii). $\operatorname{Re} q 9 x, t)$ is displayed against $x$ and $t$ when $\mu=1.6, A_{0}=$ $1.3, c_{1}:=1.3$. In (i) $\delta_{2}=-2.5, \beta=2$, (ii) $\delta_{2}=-2.5, \beta=0.7$ and (iii) $\delta_{2}=$ $-05, \beta=2$.

Figs. 6 (i)-(iii) show hybrid rhombus (diamond) waves with higher amplitude when $x>0$.While (iii) shows complex chirped waves.

(ii) The case of coupled auxiliary equations.

$$
g_{1}^{\prime}(z)=c_{1} g_{1}(z)+c_{2} g_{2}(z), \quad g_{2}^{\prime}(z)=d_{1} g_{1}(z)+d_{2} g_{2}(z) .
$$

By substituting from (32) and (29) into (13) and (14), we have

$$
\begin{gathered}
\delta_{2}=-\frac{\left(h_{2}^{2}+p_{2}^{2}\right)^{2}\left(h_{0} s_{2}-h_{2} s_{0}\right)^{4} \beta^{2}}{24 h_{2}^{4}\left(c_{2} s_{1}+d_{2} s_{2}\right)^{4} s_{0}^{4} \mu^{4}}, \quad p_{0}=\frac{h_{0} p_{2}}{h_{2}}, p_{1}=\frac{h_{1} p_{2}}{h_{2}}, \\
c_{1}=d_{2}+\frac{c_{2} s_{1}}{s_{2}}-\frac{d_{1} s_{2}}{s_{1}}, \quad \omega=k^{2} \alpha-\frac{\left(h_{2}^{2}+p_{2}^{2}\right)\left(\left(h_{2}^{2}+p_{2}^{2}\right) \beta^{2}+2 s_{2}^{2} \gamma\right)}{s^{4}} \\
-k^{3} \delta_{1}-k^{4} \delta_{2}, \quad \gamma=-\frac{\left(h_{2}^{2}+p_{2}^{2}\right)\left(h_{0}^{2} s_{2}^{2}+h_{2}^{2} s_{0}^{2}\right) \beta^{2}}{2 h_{2}^{2} s_{2}^{2} s_{0}^{2}}, \\
\sigma=\frac{1}{h_{2}^{5} s_{2}^{3}\left(c_{2} s_{1}+d_{2} s_{2}\right) s_{0}^{4}}\left(\left(-2 h_{0}^{4} p_{2}^{5} s_{2}^{4} \beta^{2}+2 h_{2}^{6} h_{0}^{2} p_{2} s_{2}^{2} s_{0}^{2} \beta^{2}\right.\right. \\
+2 h_{2}^{2} h_{0}^{2} p_{2}^{3} s_{2}^{2}\left(-2 h_{0}^{2} s_{2}+p_{2}^{2} s_{0}^{2}\right) \beta^{2}+4 h_{2}^{3} h_{0}^{2} p_{2}^{2} s_{2}^{3}\left(c_{2} s_{1}+d_{2} s_{2}\right) s_{0}^{2} \beta \mu \\
-h_{2}^{4} p_{2}\left(2 h_{0}^{4} s_{2}^{4} \beta^{2}-4 h_{0}^{2} p_{2}^{2} s_{2}^{2} s_{0}^{2} \beta^{2}+\left(c_{2} s_{1}+d_{2} s_{2}\right)^{2} s_{0}^{4} \mu^{2}\left(c_{2}^{2} s_{1}^{2} \delta_{2} \mu^{2}\right.\right. \\
\left.\left.+2 c_{2} d_{2} s_{1} s_{2} \delta_{2} \mu^{2}+s_{2}\left(\alpha-3 k \delta_{1}-6 k^{2} \delta_{2}+d_{2}^{2} \delta_{2} \mu^{2}\right)\right)\right) \\
-h_{2}^{5} s_{2}\left(c_{2} s_{1}+d_{2} s_{2}\right) s_{0}^{2} \mu\left(-4 h_{0}^{2} s_{2}^{2} \beta\right]-3 k^{2} s_{2}^{2} 2 s_{0}^{2} \delta_{1} \\
-4 k^{3} s_{2}^{2} 2 s_{0}^{2} \delta_{2}+\left(c_{2} s_{1}+d_{2} s_{2}\right)^{2} s_{0}^{2} \delta_{1} \mu^{2}+2 k s_{0}^{2}\left(2 c_{2}^{2} s_{1}^{2} \delta_{2} \mu^{2}\right. \\
\left.\left.+4 c_{2} d_{2} s_{1} s_{2} \delta_{2} \mu^{2}+s_{2}^{2}\left(\alpha+2 d_{2}^{2} \delta_{2} \mu^{2}\right)\right)\right),
\end{gathered}
$$

together with two equation for $\alpha$ and $k$ which are very lengthy to be produced here.

The solutions of the auxiliary equations are 


$$
\begin{gathered}
g_{1}(z)=\frac{1}{c_{2} s_{1}^{2}+d_{1} s_{2}^{2}} e^{\left(d_{2}-\frac{d_{1} s_{2}}{s_{1}}\right) z}\left(d_{1} s_{2}^{2} A_{0}+c_{2} s_{1}\left(-s_{2} B_{0}\right.\right. \\
\left.+e^{\frac{c_{2} s_{1} z}{s_{2}}+\frac{d_{1} s_{2} z}{s_{1}}}\left(s_{1} A_{0}+s_{2} B_{0}\right)\right), \\
g_{2}(z)=\frac{1}{c_{2} s_{1}^{2}+d_{1} s_{2}^{2}} e^{\left(d_{2}-\frac{d_{1} s_{2}}{s_{1}}\right) z}\left(c_{2} s_{1}^{2} B_{0}+d_{1} s_{2}\left(\left(-1+e^{\frac{c_{2} s_{1} z}{s_{2}}+\frac{d_{1} s_{2} z}{s_{1}}}\right) .\right.\right. \\
\left.\left.s_{1} A_{0}+e^{\frac{c_{2} s_{1} z}{s_{2}}+\frac{d_{1} s_{2} z}{s_{1}}} s_{2} B_{0}\right)\right)
\end{gathered}
$$

The solutions of (13) and (14) are

$$
\begin{gathered}
u(x, t)=\frac{1}{Q}\left(h_{0}\left(c_{2} s_{1}^{2}+d_{1} s_{2}^{2}\right)+A o e^{\left(d_{2}-\frac{d_{1} s_{2}}{s_{1}}\right) z}\left(c_{2} e^{\frac{c_{2} s_{1} z}{s_{2}}+\frac{d_{1} s_{2} z}{s_{1}}} h_{1} s_{1}^{2}\right.\right. \\
\left.+d_{1} s_{2}\left(\left(-1+e^{\frac{c_{2} s_{1} z}{s_{2}}+\frac{d_{1} s_{2} z}{s_{1}}}\right) h_{2} s_{1}+h_{1} s_{2}\right)\right)+B_{0} e^{\left(d_{2}-\frac{d_{1} s_{2}}{s_{1}}\right) z} \\
\left.\left.\left(d_{1} e^{\frac{c_{2} s_{1} z}{s_{2}}+\frac{d_{1} s_{2} z}{s_{1}}} h_{2} s_{2}^{2}+c_{2} s_{1}\left(h_{2} s_{1}+\left(-1+e^{\frac{c_{2} s_{1} z}{s_{2}}+\frac{d_{1} s_{2} z}{s_{1}}}\right)\right) h_{1} s_{2}\right)\right)\right), \\
Q=\left(c_{2} s_{1}^{2}+d_{1} s_{2}^{2}\right)\left(A_{0} e^{\left(d_{2}+\frac{c_{2} s_{1}}{s_{2}}\right) z} s_{1}+B_{0} e^{\left(d_{2}+\frac{c_{2} s_{1}}{s_{2}}\right) z} s_{2}+s_{0}\right), \\
v(x, t)=\frac{p_{2}}{h_{2}} u(x, t), \quad z=\mu x+\sigma t, p_{2}=\frac{h_{2}}{\left(h_{0} s_{2}-h_{2} s_{0}\right) \sqrt{\beta}} \\
\sqrt{\left.-\left(h 2_{0} s_{2}^{2} \beta-2 h_{2} h_{9} s_{2} s_{0} \beta+s_{0}^{2}\left(h_{2}^{2} \beta-2 c_{2} s_{1}+d_{2} s_{2}\right)^{3} \sqrt{-6 \delta_{2}} \mu^{2}\right)\right) .}
\end{gathered}
$$

The results in (35) are used to display $\operatorname{Re} q(x, t)$ against $\mathrm{x}$ and $\mathrm{t}$ in figures 8 (i), (ii) and (iii) by varying the parameters $\delta_{2}$ and $\beta$.
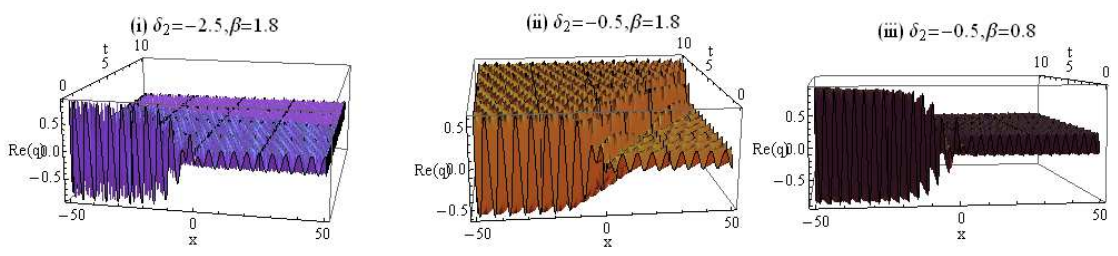

Figs. 8 (i)-(iii). $\operatorname{Re}(q)$ is displayed when $d_{1}=0.07, s_{2}=1.2, s 1:=1.4, A_{0}=$ $2.1, B_{0}=1.3, d_{2}=0.09, c_{2}=0.05, h_{2}=1.5 ; h_{0}=0.6, \beta=1.8, s_{0}=1.1, \delta_{1}=$ $1.7, h_{1}=-1.5, \mu=9$.

Figs. 8 (i) and (ii) show cascade of pulses, while (iii) shows pulses modulation which is due SPM. 


\subsection{Results and discussion}

(i) In this work, the exact solutions are obtained in the polynomial and rational functions forms. Different forms of elliptic and elementary functions are obtained. (iii) Here, it was shown that pulses propagation may take a variety of geometric structures. Some of them are novel. We mention, rhombus, complex chirped and pulses-cascade are new. Also it is found that the pulse spectrum is chaotic which agree with the results found in [34]. Relevant works on nonlocal NLSE were carried in $[35,36]$

The method used here is compared with the known methods, in the literature, as in what follows:

1- In this paper the unified method [25] was used. It unifies all known methods such as, the tanh, modified, and extended versions, the F-expansion, the exponential, the G'/G expansion method, The Kerdyashov methods .

2- On the other hand the extended unified method [27] may be sufficient to be an alternative to the use of the Lie symmetries of NLPDEs.

3 - Using the generalized unified method [28] is considered as an alternative to the Hirota bilinear method .

\section{Conclusions}

In this paper, a generalized Kundu-Eckhaus equation with extra-dispersion is presented. The colliding dynamics were investigated via a new transformation. Soliton collisions are shown to be elastic, as no rogue wave occurs. The unified method was used to determine diverse formal solutions. Abundant geometric structures of optical pulses are explored, which include:

(i) Complex chirped and W-shaped pulses; (ii) Hybrid lumps; (iii) pulses modulation induced by SPM; (iv) Rhombus (diamond) pulses.

In this new model, the wave number depends weakly on the quintic nonlinearity coefficient but it depends significantly on the coefficient of the highestorder dispersion, while as the dependence of the frequency on these parameters

is insignificant. It is expected that the results presented, here, would be helpful in experimenting higher dispersivity on the propagation of optical pulses.

The authors declare that there is no conflict of interest. 


\section{References}

[1] Serkin V. N.and Hasegawa A., Novel soliton solutions of the nonlinear Schrödinger equation Model, Phys. Rev. Lett. 85 ( 2000) 21

[2] . Ablowitz M. and Musslimani, Z. H., Integrable nonlocal nonlinear Schrodinger equation, Phys. Rev. Lett 110 (2013) 064105

[3] Guo B., Ling L. and Liu Q. P, Nonlinear Schrödinger equation: Generalized Darboux transformation and rogue wave solutions, Phys. Rev. E 85 (2012) 026607

[4] d'Avenia P., Non-radially symmetric solutions of nonlinear Schrödinger equation coupled with Maxwell equations, Advanced Nonlinear. Stud. 2 (2016) 2

[5] Carr L. D. , Charles W. C., and Reinhardt W. P., Stationary solutions of the one-dimensional nonlinear Schrödinger equation. II. Case of attractive nonlinearity, Phys. Rev. A 62 (2000) 063611

[6] Alfano R. R and Shapiro S. L., Observation of Self-Phase Modulation and Small-Scale Filaments in Crystals and Glasses, Phys. Rev. Lett. 24(1970) 592

[7] Anderson D.and Lisak M., Nonlinear asymmetric self-phase modulation and self-steepening of pulses in long optical wave guides, Phys. Rev. A 27 (1983) 1393

[8] Perry M. D., Ditmire T., and Stuart B. C., Self-phase modulation in chirped-pulse amplification, Optics. Lett., 19 (1994) 2149-2152.

[9] Tzoar, N. and Jain M, Self-phase modulation in long-geometry optical wave guides, Phys. Rev. A 23, (1981) 1266.

[10] Okawachi Y., Sharping J. E., Xu, C. and Alexander L. G. , Large tunable optical delays via self-phase modulation and dispersion, Nonl. optics fibers; 14 (2006) 12022

[11] Andresen E. R., Dudley, J. M., Oron, D., Finot, C.E, and H. Rigneault, H., Transform-limited spectral compression by self-phase modulation of amplitude-shaped pulses with negative chirp, Optics Lett. 36 (2011) No. 5 
[12] Wang X.., Yang, B., Chen Y., .and Yang, Y., Higher-order rogue wave solutions of the Kundu-Eckhaus equation, Phys. Scr. 89 (2014) 095210

[13] Baleanu D., Inc M., Yusuf A., Aliyuc A. I., Optical solitons, nonlinear selfadjointness and conservation laws for Kundu-Eckhaus equation, Chin. J. of Phys.55 (2017) 2341-2355

[14] Wanga D.-S., Guob B., Wang X., Long-time asymptotics of the focusing Kundu-Eckhaus equation with nonzero boundary conditions, J.of Diff. Equs 266 ( 2019) 5209-5253

[15] Manafian J., Akestani M. , Abundant soliton solutions for the KunduEckhaus equation via $\tan (\phi(\xi))$-expansion method, Optik 127 (2016) 55435551

[16] Bayindi C. , Rogue waves of the Kundu-Eckhaus equation in a chaotic wave field, Phys. Rev. E 93 (2016) 032201

[17] Baskonous H. M., Bulut H., On the complex structures of Kundu-Eckhaus equation via improved Bernoulli sub-equation function method, Waves in Random and Complex Media, 25 (2015) 720-728

[18] Baleanua D., Inc M., Abdullahi Yusuf A.,Aliyu A. I.,Optical solitons, nonlinear self-adjointness and conservation laws for Kundu-Eckhaus equation, Chin. J. of Phys. 55 ( 2017) 2341-2355

[19] . ui W.-T, Wazwaz A-M, Zhou,Q., LIiu W.-J., Interaction and oscillation of three soliton solutions in the variable coefficients Kundu-Eckhaus equation for dispersion management systems, Rom. J.of Phy. 64 (2019) 203

[20] . Qiu D., J. He, J., Hang, Y.and Porsezian,K., The Darboux transformation of the Kundu-Eckhaus equation, .Proc. R. Soc. A 471 (2015) 20150236

[21] Chatterjee S. K., and Khan S. N., Designing tunable narrowband parametric source in Chalcogenide-based dynamic fiber geometry, J. Opt. 23 (2021) 015503

[22] James M. Tedesco and Kevin L. Davis "Calibration of dispersive Raman process analyzers", Proc. SPIE (1999) 3537

[23] Wang H., .Boraeya M.A,Williams, L..Ballesteros, ,D. L, Vehring, R. Lowfrequency shift dispersive Raman spectroscopy for the analysis of respirable dosage forms, I. J. of Pharma.469,(2014) 197-205 
[24] Chao K, Dhakal S., ,Qin, J., .Kim, M.and Peng, Y, A , Dispersive Raman Spectral Imaging System for Food Safety and Quality Evaluation, Appl. Sci. 8 (2018) 431

[25] Abdel-Gawad H. I., Towards a unified method for exact solutions of evolution equations. An application to reaction diffusion equations with finite memory transport." J of Stat. Phys. 147 ( 2012) 506

[26] Abdel-Gawad H. I.,Solutions of the generalized transient stimulated Raman scattering equation. Optical pulses compression, Optik 230 (2021) 166314.

[27] Abdel- Gawad H.I, Elazab N. S.and M. Osman. "Exact solutions of space dependent Korteweg-de Vries equation by the extended unified method." J. of the Phys.Soc. of Japan 82 (2013) 044004

[28] Abdel-Gawad H.I., Biswas A. Multi-soliton solutions based on interactions of basic traveling waves with an applications to the non local Boussinesq equation. Acta Phys. Pol. B 47 (2016) 1101-1112

[29] Abdel-Gawad H.I., Tantawy M., A novel model for lasing cavities in the presence of population inversion: Bifurcation and stability analysis, Chaos, Solitons and Fractals 144 (2021) 110693

[30] Ma Y.L, Interaction and energy transition between the breather and rogue wave for a generalized nonlinear Schrödinger system with two higher-order dispersion operators in optical fibers. Nonlinear Dyn. 97 (2019) 95

[31] Qiu D., Cheng W., N-fold Darboux transformation of the two-component Kundu-Eckhaus equations and non-symmetric doubly localized rogue waves, Eur. Phys. J. Plus 13 (2020) 135

[32] 1 Manafian J., Amin M., Murad S., Alizadeh A., Jafarmadar S., M-lump, interaction between lumps and stripe solitons solutions to the $(2+1)$ dimensional KP-BBM equation Eur. Phys. J. Plus 135 (2020) :167

[33] Kundu A.,"Landau-Lifshitz and higher-order nonlinear systems gauge generated from nonlinear Schrödinger-type equations", J. of Math. Phys., 25 (1984) 3433-3438

[34] Bayındı C., Rogue wave spectra of the Kundu-Eckhaus equation, Phys. Rev. E 93, (2016) 062215 
[35] Ma W. X. and Chen M., Direct search for exact solutions to the nonlinear Schrödinger equation, Appl. Mathe.and Comput. 215 (2009) 2835-2842

[36] Ma W. X., Inverse scattering for nonlocal reverse-time nonlinear Schrödinger equations, Appl. Mathe.Lett., 102 (2020) 106161 
(i)

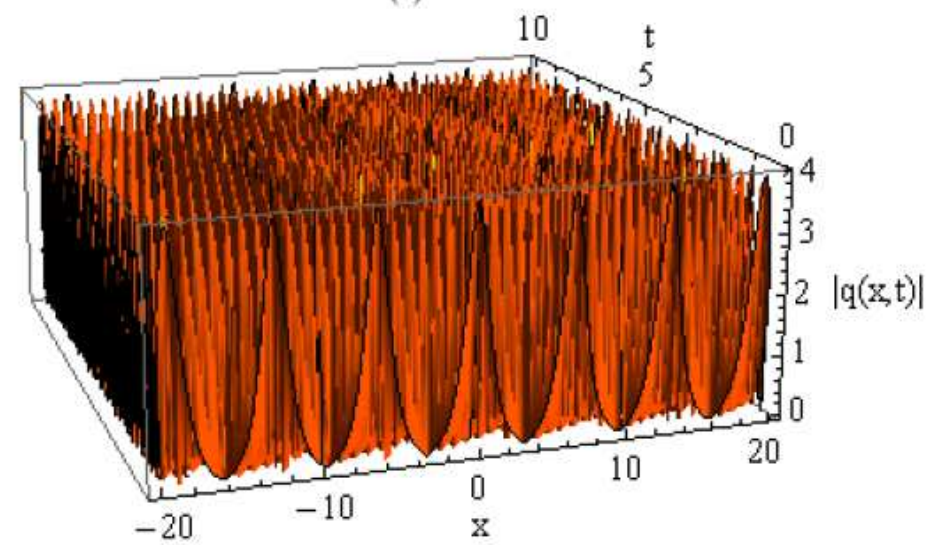

(ii)

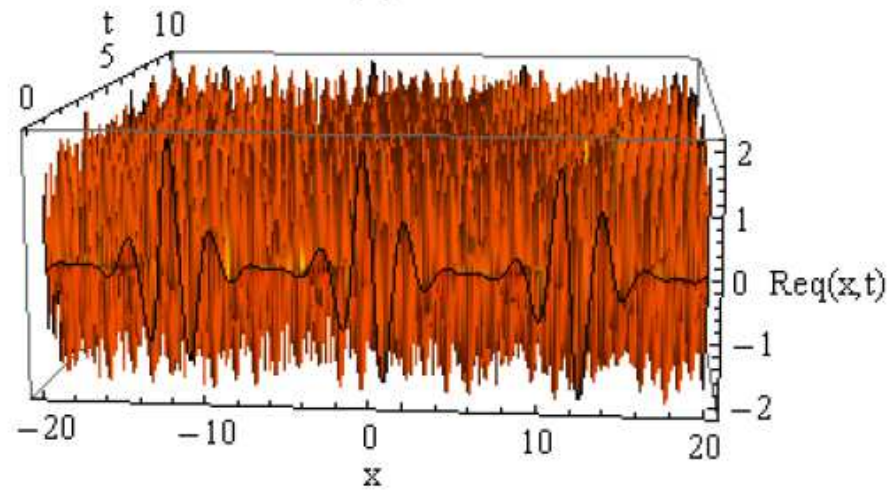

Figure 1

(i) and (ii) when $\mathrm{m}:=0: 999 ; \delta 2=-0.5 ; \delta 1=2.5 ; \mathrm{h} 1=0.6, \beta=0.5 ; \mu=0: 8, \gamma=1.1$. Fig. (i) shows hybrid $\mathrm{W}$ shaped waves while (ii) shows complex chirped waves.
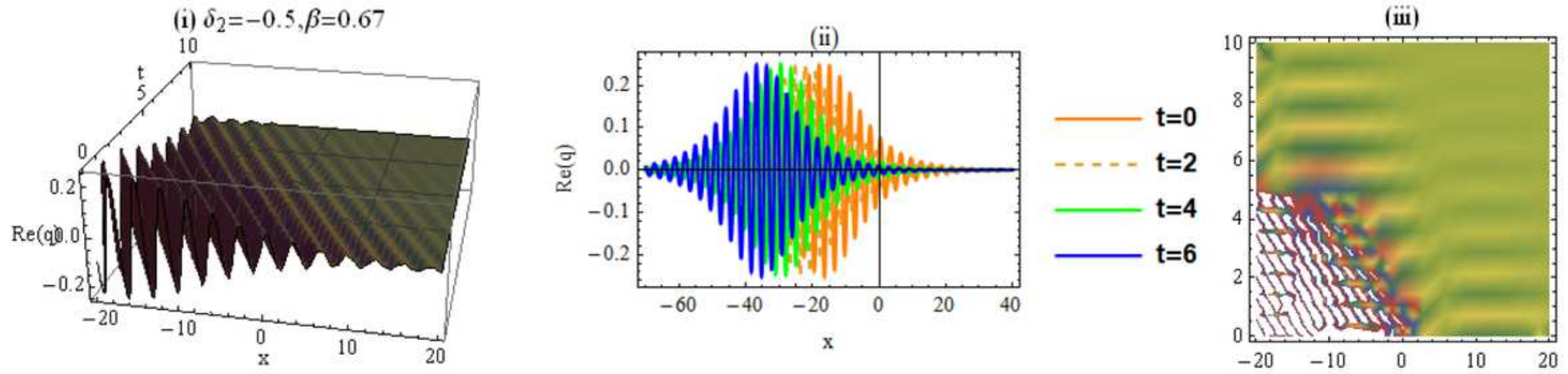

Figure 2

(i)-(iii). $\operatorname{Req}(x, t)$ is displayed when $p 1=-0.6, \delta 2=-0.5, \beta=0.67, a=1.1, b=3.5, \mu=0.4, A 0=1.6, y=0.4$, $\delta 1=1.8$. In (i) the $3 \mathrm{D}$ plot, (ii) against $x$ for different values of $t$ and (iii) the contour plot.
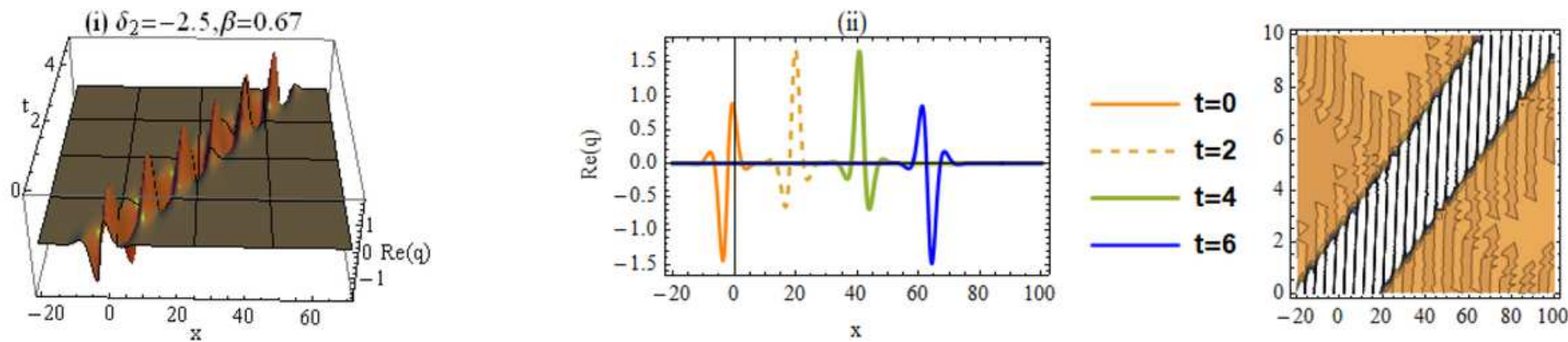

Figure 3 
(i)-(iii). Req( $(x, t)$ is displayed for the same caption as in Figs.2 (i)-(iii).
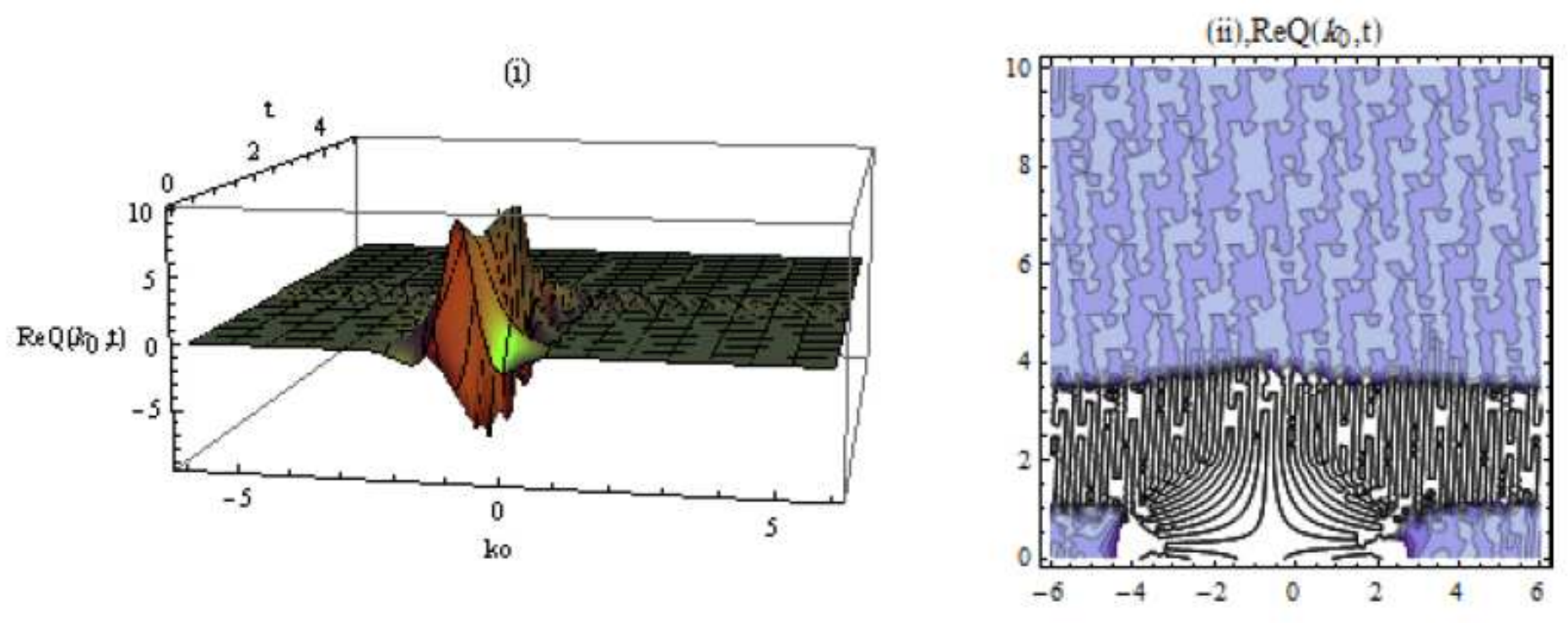

Figure 4

(i) and (ii). With relevance to (22), the spectrum is displayed when $\mathrm{p} 1=-0.6, \mathrm{a}=1.1, \mathrm{~b}=2.5, \mu=0.5, \mathrm{~A} 0=$ $1.6, Y=0.4, \delta 1=1.8, \beta=0.7, \delta 2=-2.5$. The $3 \mathrm{D}$ and contour plots are shown respectively.

(i) $\delta_{2}=-2.5, \beta=3$

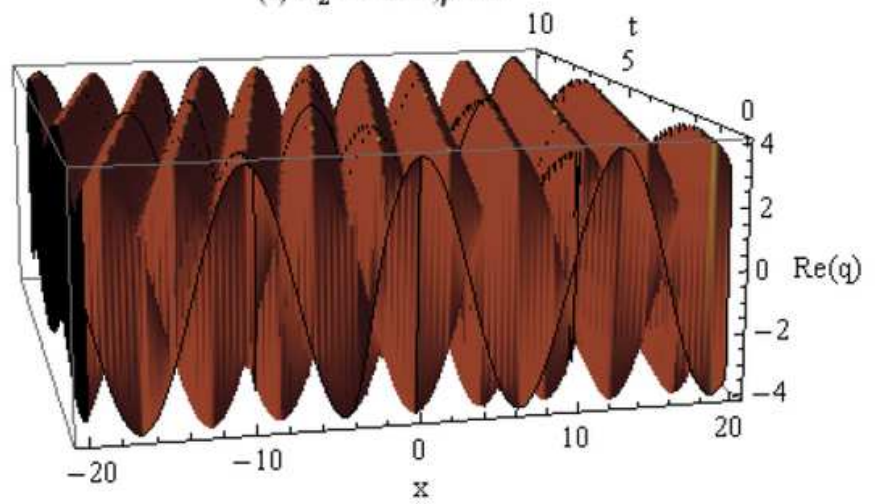

(ii)

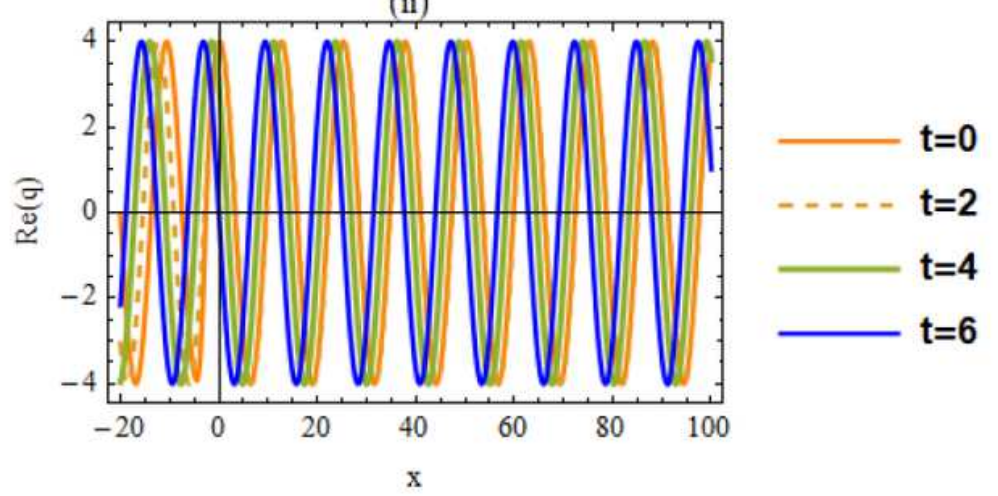

Figure 5

(i) and (ii). When $\mathrm{h} 1=0.6, \delta 2=-2.5, \beta=3 ; \mathrm{c} 0=1.6, \mathrm{c} 1=3.1, \mathrm{c} 2=-1.5, \mu=0.5, \mathrm{~A} 0=1.6, \delta 1=1.8, \mathrm{k}=0.5$, h0 $=2.5$. Fig. (i) shows periodic rhombus (diamond) waves in space and time, while (ii) shows periodic waves in space and time. 


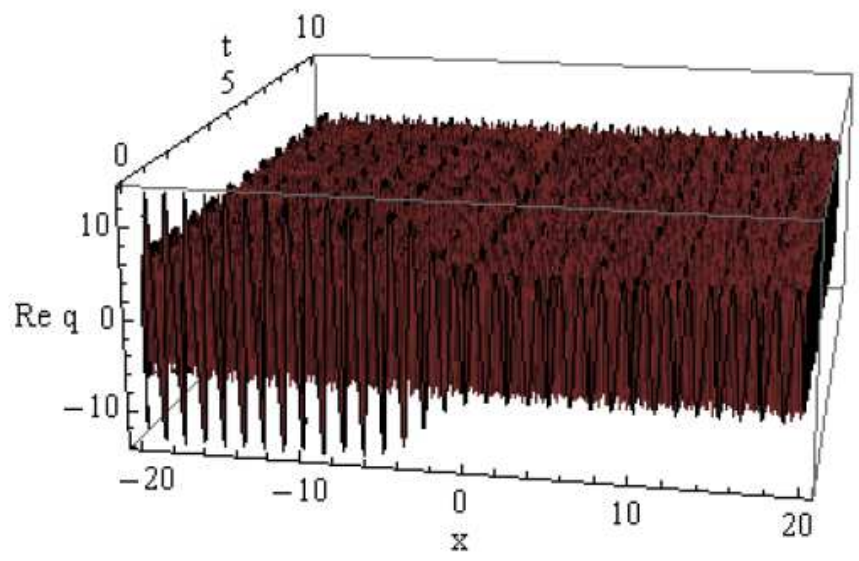

(ii)

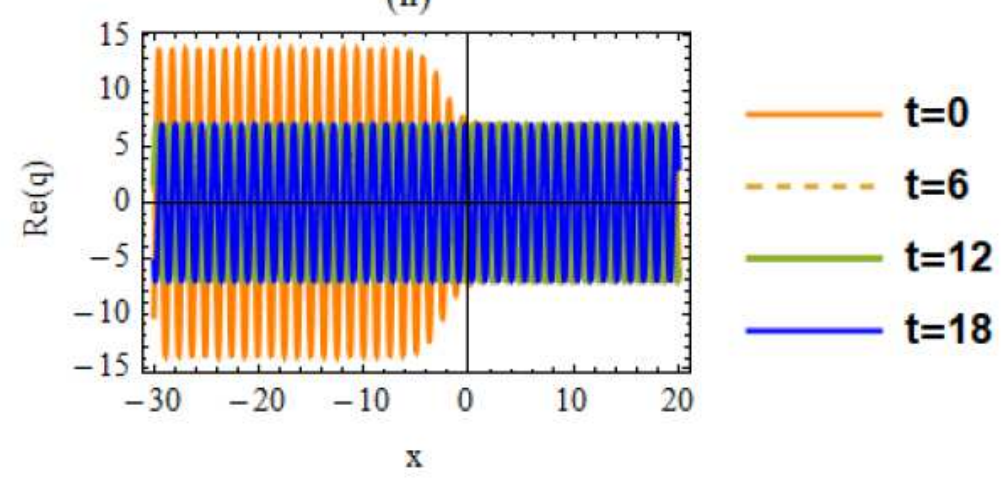

Figure 6

(i) and (ii0. When $A 0=1.1, p 1=0.9, \delta 2=-2.5, \beta=3, p 2=-1.7, \mu=0.3$. Fig. (i) shows hybrid chirped waves on $\mathrm{x}<0$ and periodic $\mathrm{M}$-shaped waves on $\mathrm{x}>0$, while (ii) shows solitary wave modulation, which is due to SPM.

(i) $\delta_{2}=-2.5, \beta=2$

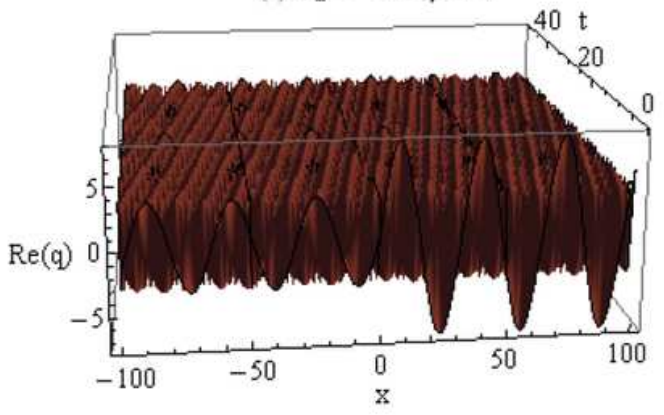

(ii) $\delta_{2}=-2.5, \beta=0.7$

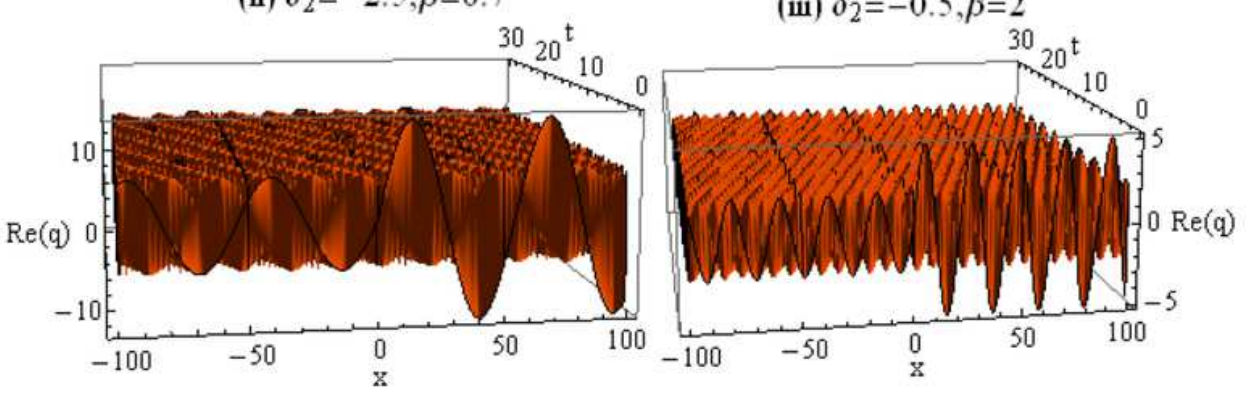

Figure 7

(i)-(iii). Req9x; t) is displayed against $x$ and t when $\mu=1.6, A 0=1.3, \mathrm{c} 1:=1.3$, In (i) $\delta 2=-2.5, \beta=2$, (ii) $\delta 2=$ $-2.5, \beta=0.7$ and (iii) $\delta 2=-05, \beta=2$.
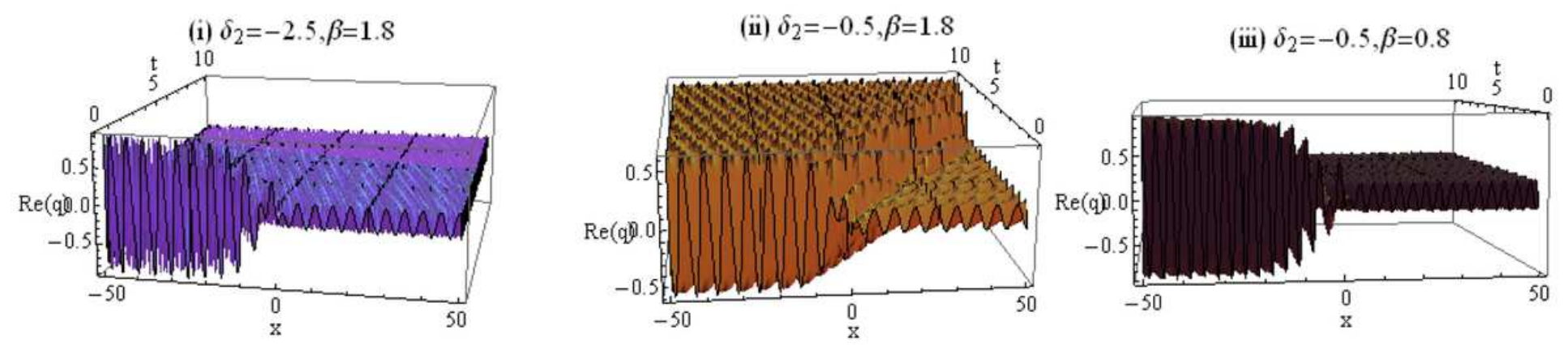

\section{Figure 8}

(i)-(iii). $\operatorname{Re}(\mathrm{q})$ is displayed when $\mathrm{d} 1=0.07, \mathrm{~s} 2=1.2, \mathrm{~s} 1:=1,4, \mathrm{~A} 0=2.1, \mathrm{~B} 0=1.3, \mathrm{~d} 2=0.09, \mathrm{c} 2=0.05, \mathrm{~h} 2=$ $1.5, \mathrm{~h} 0=0.6, \beta=1.8, \mathrm{~s} 0=1.1, \delta 1=1.7, \mathrm{~h} 1=-1.5, \mu=9$. (i) and (ii) show cascade of pulses, while (iii) 
shows pulses modulation which is due SPM. 